\title{
Anabases
}

ANABASES Traditions et réceptions de l'Antiquité

$4 \mid 2006$

Varia

\section{Nicolas Rouvière, Astérix ou les lumières de la civilisation, Préface de P. Ory}

Olivier Devillers

\section{OpenEdition}

Journals

Édition électronique

URL : http://journals.openedition.org/anabases/2871

DOI : 10.4000/anabases.2871

ISSN : 2256-9421

Éditeur

E.R.A.S.M.E.

Édition imprimée

Date de publication : 1 octobre 2006

Pagination : 319-321

ISSN : 1774-4296

\section{Référence électronique}

Olivier Devillers, « Nicolas Rouvière, Astérix ou les lumières de la civilisation, Préface de P. Ory », Anabases

[En ligne], 4 | 2006, mis en ligne le 01 mai 2012, consulté le 22 septembre 2020. URL : http://

journals.openedition.org/anabases/2871 ; DOI : https://doi.org/10.4000/anabases.2871

Ce document a été généré automatiquement le 22 septembre 2020.

(c) Anabases 


\title{
Nicolas RouviÈre, Astérix ou les lumières de la civilisation, Préface de P. Ory
}

\author{
Olivier Devillers
}

\section{RÉFÉRENCE}

Nicolas RouvièRE, Astérix ou les lumières de la civilisation, Préface de P. Ory, (Coll. Partage du savoir), Paris, PUF, 2006, XIV + 229 p., 15,5 x 24.

25 euros / ISBN 213055265X.

1 L'idée forte qui anime et porte cet ouvrage, issu d'une thèse de doctorat, est que « tout ce qui se dit dans Astérix ne saurait être indifférent » (p. 221). Cette conviction justifie que soient analysés et pourvus de sens les moindres détails de la série. Il s'ensuit une relecture très stimulante de celle-ci, selon une méthode et une problématique ( $c f$. «Introduction » p. 1-13) qui sont profondément, et même radicalement, littéraires. Les spécialistes de la littérature ancienne, notamment, se réjouiront de voir appliquée à une bande dessinée une démarche qui leur est familière : celle qui consiste à traquer la signification de chaque élément du texte et, au-delà de la recherche des intentions conscientes d'un auteur, à s'interroger sur ses intuitions créatrices. En ce sens, beaucoup des remarques qui sont amassées ici, dans la mesure où elles s'appuient sur le texte (et l'image), semblent en elles-mêmes irréfutables (par ex., la récurrence du motif de la marmite ou du chaudron). Par contre, leur mise en système ne convainc pas toujours. Une raison en est le recours fréquent à un symbolisme empreint de subjectivité ; les idées de coupure, de limite... sont particulièrement mises en avant et leur application semble parfois davantage tenir à une construction de l'Auteur de l'étude que traduire des intentions, même inconscientes, des auteurs de la série, par exemple à propos de la potion magique («désenlacer le corps et l'image») ou des sangliers (« sang-lier » comme emblème généalogique)... On regrettera aussi l'absence 
d'une réflexion sur le genre comique auquel ressortit la série. Les impératifs de celui-ci, son ambiguïté, le fait que maintes péripéties scénaristiques n'ont de raison d'être qu'en fonction de calembours rendent difficile une analyse globale d'un corpus constitué de 26 albums (seules sont considérées les collaborations entre Uderzo et Goscinny). Le but premier d'Astérix est, sinon d'atteindre le succès commercial, du moins d'offrir un divertissement et, s'il y a une dimension politique, celle-ci n'est que collatérale, une constatation qui amène à redimensionner certaines des conclusions proposées dans cette étude. Ceci vaut en particulier, à mon sens (mais il s'agit là d'une sensibilité de lecteur), pour l'analyse du village gaulois ; l'explication de celui-ci selon une structure en étoile, en référence au nom même d'Astérix, paraît pécher par excès de subtilité, de même qu'il est a priori difficile de réprimer un sourire lorsqu'on lit que les poissons d'Ordralfabétix incarnent la mère ou qu'Assurancetourix a pour fonction de « rappeler l'existence d'un vide logique pour cimenter le lien social» (p. 162)... Par contre, des observations portant sur des aspects plus ponctuels du récit (par ex. les Goths...) ou sur des éléments « secondaires » de celui-ci (les Romains) se révèlent plus convaincantes, ainsi ce qui regarde la présentation de l'absolutisme romain et égyptien comme infantilisant.

2 L'éclairage de l'ouvrage par le contexte de rédaction de la série et par la personnalité de ses auteurs - une démarche aussi qui est partie intégrante de l'explication de texte est également développé, apportant à travers l'évocation de la jeunesse des auteurs, de la vie du journal Pilote, du climat politique de la France des années '60 et '70 ou encore la comparaison avec d'autres écrits de Goscinny (le scénario du film Le Viager ou son roman Tous les visiteurs à terre)... des éléments explicatifs intéressants. Mais là encore, au moment de dégager un système, il apparait qu'il est plus facile de dire ce qu'Astérix n'est pas que ce qu'il est (la sensibilité «mendésienne " n'emporte pas totalement l'adhésion) et le sentiment qui demeure est celui de la complexité de la série, ce que traduit peut-être un peu malgré elle la formule d' "individualisme humaniste" (p. 217). Par ailleurs, à part quelques pages sur le mariage de Goscinny, la vie privée des auteurs est totalement évacuée; il est difficile de croire que, comme l'a fait leur vie professionnelle, elle n'a pas joué dans l'élaboration de certains épisodes.

3 En somme, le principal reproche que l'on adressera à ce livre est de vouloir appliquer des grilles de lecture systématiques à un corpus où l'humour et l'humeur du moment interviennent assurément beaucoup, et dans des sens parfois divergents. L'analyse est aussi fortement focalisée sur Astérix et les allusions aux autres séries de Goscinny sont rares. À la page 176, l'Auteur semble proposer San Antonio comme objet d'enquête, mais nous serions pour notre part curieux de voir comment il analyserait l'univers de Lucky Luke, série de laquelle les jeux de mots (refusés par Morris) sont bannis. De même, il aurait été intéressant de se pencher sur la série d'aviation Michel Tanguy, collaboration - abandonnée en 1967 et qui n'est pas mentionnée ici - entre Uderzo et J.-M. Charlier, cité à plusieurs reprises à propos des pirates. Pour autant, nous n'avons pas affaire à une étude-gadget, mais à un vrai travail de réflexion et de recherche qui engage à considérer comme une création littéraire au sens large une forme ludique de réception de l'Antiquité qui n'est pas négligeable, ni - cela est évident - en termes de notoriété, ni en termes de survie, dans la mesure où elle se prête à la constitution d'univers " fictifs » ancrés dans un imaginaire ouvert à de multiples lectures. En cela, ce livre nous convainc qu'Astérix, à travers l'utopie d'un village gaulois (et au-delà de tout recours à l'anachronisme comme ressort comique), est aussi un discours - construit à la 
fois par ses auteurs, par sa dynamique propre et par ses lecteurs - sur notre propre société.

\section{AUTEURS}

\section{OLIVIER DEVILLERS}

Université Bordeaux III - Michel de Montaigne oldevilliers@wanadoo.fr 